Review Article

\title{
The Relationship between Helicobacter pylori Infection of the Gallbladder and Chronic Cholecystitis and Cholelithiasis: A Systematic Review and Meta-Analysis
}

\author{
Liang Wang, ${ }^{1,2}$ Junyin Chen, ${ }^{1,3}$ Wenxi Jiang, ${ }^{1}$ Li Cen, ${ }^{1}$ Jiaqi Pan, ${ }^{1}$ Chaohui Yu, ${ }^{1}$ Youming Li, \\ Weixing Chen, ${ }^{1}$ Chunxiao Chen $\left(10,{ }^{1}\right.$ and Zhe Shen $(1){ }^{1}$ \\ ${ }^{1}$ Department of Gastroenterology, The First Affiliated Hospital, College of Medicine, Zhejiang University, Hangzhou, China \\ ${ }^{2}$ Endoscopy Center, Cangzhou Central Hospital of Hebei Province, Cangzhou, Hebei Province, China \\ ${ }^{3}$ Department of Gastroenterology, The Affiliated Hospital, Shaoxing College of Arts and Sciences, Shaoxing, \\ Zhejiang Province, China
}

Correspondence should be addressed to Chunxiao Chen; cxcenli@sina.cn and Zhe Shen; sz8239@zju.edu.cn

Received 12 September 2020; Revised 4 November 2020; Accepted 28 November 2020; Published 6 January 2021

Academic Editor: Yousuke Nakai

Copyright (C) 2021 Liang Wang et al. This is an open access article distributed under the Creative Commons Attribution License, which permits unrestricted use, distribution, and reproduction in any medium, provided the original work is properly cited.

Helicobacter pylori (H. pylori) is proved to be the main pathogenic agent of various diseases, including chronic gastritis, gastric ulcer, duodenal ulcer, and gastric cancer. In addition, chronic cholecystitis and cholelithiasis are common worldwide, which are supposed to increase the total mortality of patients. Epidemiologic evidence on the relationship between $H$. pylori infection of the gallbladder and chronic cholecystitis/cholelithiasis still remains unclear. We conducted a systematic review and meta-analysis of overall studies to investigate the relationship between $H$. pylori infection of the gallbladder and chronic cholecystitis/cholelithiasis. Two researchers searched PubMed, Embase, and Cochrane Library databases to obtain all related and eligible studies published before July 2020. The pooled odds ratios (ORs) and corresponding 95\% confidence intervals (CIs) were calculated by the randomeffects model. Subgroup analysis, heterogeneity, publication bias, and sensitivity analysis were also conducted. Twenty studies were included in the meta-analysis, involving 1735 participants and 1197 patients with chronic cholecystitis/cholelithiasis. Helicobacter species infection of the gallbladder was positively correlated with increased risk of chronic cholecystitis and cholelithiasis, especially $H$. pylori $\left(\mathrm{OR}=3.05 ; 95 \% \mathrm{CI}, 1.81-5.14 ; I^{2}=23.5 \%\right)$. Besides, country-based subgroup analysis also showed a positive correlation between the gallbladder H. pylori positivity and chronic cholecystitis/cholelithiasis risk. For Asian and non-Asian country studies, the ORs were 4.30 (95\% CI, 1.76-10.50; $\left.I^{2}=37.4 \%\right)$ and $2.13\left(95 \% \mathrm{CI}, 1.23-3.70 ; I^{2}=0.0 \%\right)$, respectively. The association was more obvious using the bile sample and urease gene primer. In conclusion, this meta-analysis provided evidence that there is a positive correlation between $H$. pylori infection in the gallbladder and increased risk of chronic cholecystitis and cholelithiasis.

\section{Introduction}

Helicobacter pylori (H. pylori) is a Gram-negative, spiralshaped, and microaerophilic microorganism, which is proved to be the main pathogenic agent in the occurrence and development of chronic gastritis, gastric ulcer, and duodenal ulcer [1]. Furthermore, H. pylori is also associated with an increased risk of gastric malignant lymphoma of the mucosa-associated lymphoid tissue (MALT) as well as gastric adenocarcinoma [2-4].
Chronic cholecystitis and cholelithiasis are quite widespread worldwide. Geography and especially ethnicity play an important role in the prevalence of gallstone diseases. The prevalence rate in the west is higher than that in the east, ranging from $4 \%$ to $74 \%$ [5]. Recently, some studies indicated that people with gallstone diseases had increased total mortality and cardiovascular disease and cancer mortality $[6,7]$. Besides, since chronic inflammation is caused by repeated trauma to the gallbladder mucosa and DNA damage led by high or abnormal bile acid exposure, 
gallstones are closely related to the development of hepatobiliary cancers, including gallbladder cancer [8]. Therefore, chronic cholecystitis and cholelithiasis constitute a serious health problem, which brings a great medical burden to the society.

Since Chang et al. [9] incidentally discovered H. pylori in the gallbladder's mucosa of a patient with cholecystitis in 1996, more and more studies have detected various Helicobacter species including $H$. pylori, $H$. bilis, $H$. hepaticus, $H$. pullorum, and $H$. ganmani in the gallbladder tissue, gallstone, and bile taken from the gallbladder [10, 11]. Among these various Helicobacter species, $H$. pylori is the commonest one. The high prevalence of $H$. pylori in the group of patients with chronic cholecystitis raises questions concerning about the role of $H$. pylori infection in chronic cholecystitis. During the formation of gallstones, there are many etiological factors. Also, among them, infection, inflammation, and the immune system play a vital role [12]. Recently, increasing studies pointed that the gut microbiome participated in gallstone formation by reducing bile acid metabolism and affecting the dynamic equilibrium $[13,14]$. H. pylori is one of them.

Therefore, a series of studies have been carried out to find the relationship between gallbladder $H$. pylori infection and chronic cholecystitis/cholelithiasis [15-34]. Karagin et al. [28] supported the role of $H$. pylori in the development of cholecystitis in humans. Also, Dar et al. [32] pointed an association between the presence of $H$. pylori and hepatobiliary stone diseases. However, in a study conducted by Fallone et al. [35], they found no $H$. pylori present in any of the patients diagnosed with gallstones, thus raising doubt as to the possible association between $H$. pylori and gallstone diseases.

Consequently, the relationship between $H$. pylori infection in the gallbladder and cholecystitis/cholelithiasis still remains unclear, with controversial results reported in various studies. We perform this meta-analysis aiming to review data from related studies to evaluate the potential association between $H$. pylori infection in the gallbladder and chronic cholecystitis/cholelithiasis.

\section{Methods}

This systematic review and meta-analysis were conducted based on the Meta-Analysis of Observational Studies in Epidemiology (MOOSE) statement guidelines [36].

2.1. Search Strategy. We searched for relevant studies from PubMed, Embase, and Cochrane databases. The last search was in July 2020. The retrieval strategy used was as follows: ((Helicobacter pylori) or (Campylobacter pylori) OR (H. pylori) OR (HP) OR (Helicobacter) OR (Helicobacter bilis) OR (Helicobacter hepaticus) OR (Helicobacter pullorum) OR (H. bilis) OR (H. hepaticus) OR (H. pullorum) OR (H. ganmani) OR (Helicobacter species) OR (Helicobacter sp.) OR (Helicobacter genus) OR Campylobacter OR (Campylobacter infection) OR Campylobacteriosis OR (Helicobacter pylori infection) OR (Helicobacter infection)
OR (pylori) OR (enterohepatic Helicobacter spp.) OR (Campylobacter spp.)) AND (cholelithiasis or cholecystolithiasis or gallstone* OR gall* stone* OR (gallbladder AND stone*) OR (gallbladder AND cholelith) OR (gallbladder AND lithiasis) OR bilestone* OR (bile AND stone*) OR (bile AND lithiasis) OR (bile AND cholelith) OR (biliary AND calculus) OR (biliary AND stone*) OR (biliary AND cholelith) OR (biliary AND lithiasis) OR cholecystitis). We also supplemented eligible studies from the references in the review articles by manual retrieval.

2.2. Eligibility Criteria. The inclusion criteria are as follows: (1) original cross-sectional studies, cohort studies, or casecontrol studies which are about the relationship between chronic cholecystitis/cholelithiasis and Helicobacter species infection of the gallbladder, (2) papers in which the method of detecting Helicobacter species infection was polymerase chain reaction (PCR), (3) papers that provide sufficient information to calculate odds ratios (ORs) and 95\% confidence intervals (CIs), and (4) published in English as a full paper before July 2020.

The exclusion criteria are as follows: (1) reviews, metaanalysis, letters, commentaries, case reports, and animal studies, (2) studies that had no control group or inappropriate control group, and (3) duplicate study samples.

2.3. Data Extraction and Quality Assessment. Two investigators extracted the following data from each study in a standardized manner, independently: first author's name, publication year, country, study population and demographic data (e.g., gender and age), biliary diseases, the detection method for Helicobacter species, sample, specific primer, Helicobacter species, as well as the number of cases and controls.

The quality of each study included was also independently assessed by two investigators according to the 9-star Newcastle-Ottawa quality assessment scale [37], the content of which is as follows: (1) the selection of cases and controls, (2) the comparability of cases and controls, and (3) the assessment of exposure.

Any discrepancies on data extraction or quality assessment were resolved through discussion.

2.4. Statistical Analysis. All statistical analyses were conducted with Stata statistical software (version 12.0; College Station, Texas 77845, USA) by two authors, independently. Also, we used a random-effect model to calculate the summarized ORs with 95\% CIs considering the diversity among various studies due to different study designs, methodologies, and populations.

The standard chi-square test was performed to assess statistical heterogeneity between studies, and the result was quantified by $I^{2}$. Values of $I^{2}<25 \%, 25-50 \%$, and $>75 \%$ indicate low, medium, and high heterogeneity, respectively. When highly heterogeneous outcome was observed, sensitivity analysis was performed to investigate the influence of the individual study and the stability of results. 
Because of the regional differences in the prevalence of H. pylori infection [38], we also conducted a subgroup analysis of studies from Asian and non-Asian countries in order to reduce regional interference in the study. We also performed subgroup analyses of samples and primers for detecting H. pylori.

2.5. Assessment of Bias and Sensitivity Analysis. Publication bias was assessed by funnel scatter-plots, Begg's adjusted rank correlation, and Egger's regression asymmetry tests. Publication bias will lead to asymmetry in funnel plots. Also, for Begg's and Egger's test, $P<0.05$ was considered to indicate potential publication bias.

As for sensitivity analysis, leave-one-out sensitivity and stratified analysis were also carried out to investigate the influence of the individual study and the stability of results.

\section{Results}

3.1. Description of the Studies. The search strategy yielded 826 publications, of which 202 were from PubMed, 611 were from Embase, and 13 were from the Cochrane Library. Of these records, 193 were excluded as duplicate articles and 494 were excluded as irrelevant on the basis of title and abstract. 139 were reviewed for detailed assessment. Of these publications, 119 were excluded from our final analysis, and the reasons are listed in Figure 1. Besides, 2 articles by Bulajic et al. $[16,17]$ were included in our meta-analysis due to the independence of the sample. Eventually, 20 studies, published between 2000 and 2018, were included in our metaanalysis.

The main characteristics of the included studies are listed in Table 1. The selected 20 studies included a total size of 1735 participants, of which 1197 were for the case group and 538 were for the control one. Of these studies, 11 of them were conducted in Asia (Japan, India, Thailand, Iran, Pakistan, and Russia) and 9 studies were conducted in nonAsian areas (German, Serbia, Brazil, Greece, Sweden, New Zealand, and Netherlands). There were totally 5 species of Helicobacter species including $H$. pylori, $H$. bilis, $H$. hepaticus, H. pullorum, and H. ganmani in our study. $H$. pylori was the commonest species which was identified in 17 studies. H. bilis was reported in 2 studies. H. hepaticus, $H$. pullorum, and H. ganmani each were reported in 1 study. Among the included studies, 12 studies used bile as sample, 5 studies used the gallbladder as sample, 2 studies used bile and the gallbladder as sample, and only 1 study used gallstone as sample. The specimens of bile, gallbladder tissue, and gallstone were obtained during cholecystectomy, endoscopic retrograde cholangiopancreatography (ERCP), percutaneous transhepatic cholangio-drainage, endoscopic papillotomy, and regular operation of gastrectomy or hepatectomy. Polymerase chain reaction (PCR) was used to detect Helicobacter species infection, and the positive rate is $344 / 1735$. The primer of target genes in the included studies varied. The $16 \mathrm{~S}$ rRNA gene was the most commonly used primer and had been used in 12 studies. Urease gene was used as primer in 3 studies, and $26 \mathrm{kDa}$ species-specific protein gene was used in 1 study. 3 other studies used two primers in combination.

3.2. Study Quality. Each study chosen for this meta-analysis was carefully assessed according to the Newcastle-Ottawa scale assessment. All studies obtained high scores, defined as $\geq 6$ stars (Table 1).

3.3. The General Analysis. Overall, as shown in Figure 2, the pooled OR for patients with chronic cholecystitis/cholelithiasis compared with participants without chronic cholecystitis/cholelithiasis was 3.15 (95\% CI, 2.01-4.93), indicating a potential positive association between gallbladder Helicobacter species infection and chronic cholecystitis/cholelithiasis risk. Also, there was low heterogeneity $\left(I^{2}=20.1 \%, P=0.205\right)$.

Besides, we found a higher gallbladder $H$. pylori infection rate in the chronic cholecystitis/cholelithiasis group than that in the control group in 17 studies that focused on H. pylori $(\mathrm{OR}=3.05 ; 95 \% \mathrm{CI}, 1.81-5.14)$ (Table 2). For other Helicobacter species, studies concerned were limited (Table 2). In addition to $H$. pylori, a higher $H$. hepaticus infection rate in the chronic cholecystitis/cholelithiasis than that in the controls was also observed $(41.7 \%$ vs. $12.5 \%$, $P=0.007)$. Although the prevalence of $H$. bilis was also higher in chronic cholecystitis/cholelithiasis $(21.3 \%$ vs. $10.3 \%, P=0.273$ ), the results had no statistically significant difference. $H$. pullorum and $H$. ganmani infection rates were low in both cases and controls $(6.0 \%$ vs. $0.0 \%, P=0.073$; $1.8 \%$ vs. $0.0 \%, P=0.914$, respectively).

3.4. Subgroup Analysis for $H$. pylori Infection. For $H$. pylori infection, subgroup analyses were conducted according to country, sample, and specific primer in Figure 3. Compared with overall results, there were no significant differences in subgroup studies. Gallbladder H. pylori infection was also positively correlated with the risk of chronic cholecystitis/ cholelithiasis in both Asian and non-Asian groups. The ORs for Asian and non-Asian country studies were 4.30 (95\% CI, $\left.1.76-10.50 ; \quad I^{2}=37.4 \%\right)$ and $2.13 \quad(95 \%$ CI, $1.23-3.70$; $\left.I^{2}=0.0 \%\right)$, respectively. In bile samples, the pooled OR was 3.78 (95\% CI, 1.63-8.79), indicating a significantly higher detection rate in the chronic cholecystitis/cholelithiasis patients than that in the control group. In gallbladder samples, although the pooled OR was 2.03 (95\% CI, $0.65-6.40)$, the result had no statistically significant difference $(P=0.226)$. Only 1 study used the gallstone as the sample, and the result had no statistically significant difference $(P=0.377)$. The ORs of $16 \mathrm{~S}$ rRNA gene and urease gene were 2.27 (95\% CI, 1.25-4.13) and 10.18 (95\% CI, $2.31-44.80$ ), respectively. Only 1 study used $26 \mathrm{kDa}$ protein gene.

3.5. Assessment of Bias and Sensitivity Analysis. As shown in Figure 4, funnel plot is relatively symmetrical, suggesting no strong evidence for publication bias. In addition, there was 

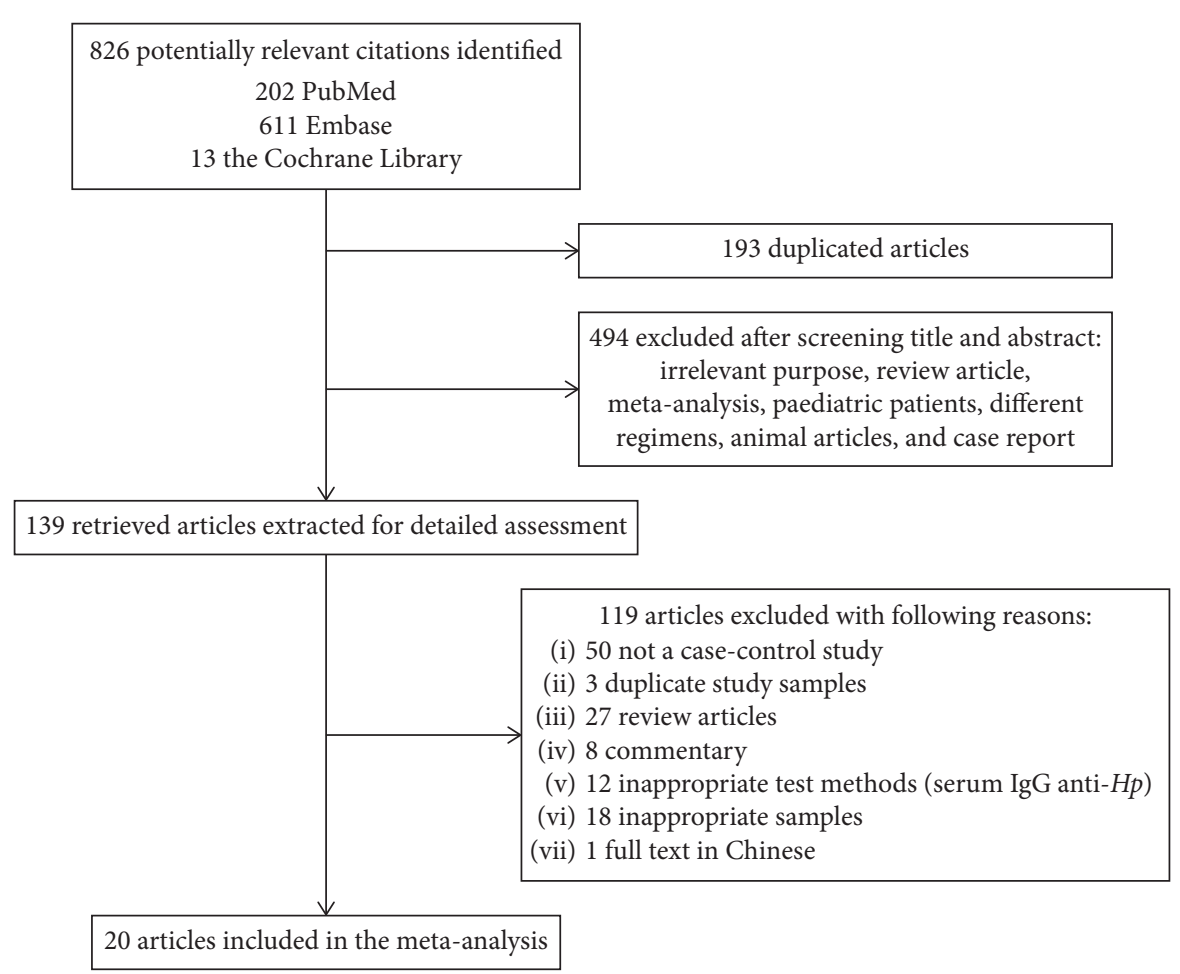

FIgURE 1: Flow diagram of the systematic review of the literature.

no evidence for bias using either Begg's method $(P=0.974)$ or Egger's weighted regression method $(P=0.268)$.

All the results remained significant when leave-one-out sensitivity analyses were conducted, indicating the stability of results for this meta-analysis.

\section{Discussion}

Since Helicobacter species was detected from the gallbladder tissue, gallstone, and bile taken from the gallbladder, numerous studies have shown that Helicobacter species, especially $H$. pylori, may contribute to the formation of cholesterol gallstones [39]. However, research studies conducted so far have failed to establish a definite relationship between $H$. pylori infection and gallstone diseases. Our systematic review and meta-analysis is to assess the relationship between $H$. pylori infection in the gallbladder and chronic cholecystitis/cholelithiasis.

Overall, our research showed that the prevalence of Helicobacter species in the chronic cholecystitis/cholelithiasis group was significantly higher than that in the control group $(24.40 \%$ vs. $8.55 \%, P=0.000)$, suggesting a potential positive correlation between Helicobacter species infection in the gallbladder and increased risk of chronic cholecystitis/ cholelithiasis. Likewise, a higher gallbladder $H$. pylori infection rate was found in the chronic cholecystitis/cholelithiasis group than that in the control group $(23.70 \%$ vs. $7.23 \%, P=0.000)$. Due to the small size of relevant studies, there was no significantly positive correlation between other Helicobacter species infection in the gallbladder with gallstone diseases except for $H$. hepaticus.
People in countries with lower socioeconomic status are more likely to be infected with $H$. pylori. Previous studies [38] reported that the prevalence of H. pylori in Asia, Africa, and South America is higher than that in North America, Western Europe, and Australia, which may influence $H$. pylori infection rate in the gallbladder. So we divided the subgroups according to whether they were Asian countries or not. This positive association still persisted both in Asian or non-Asian countries. Also, the OR of Asian countries is quite higher than that of non-Asian countries (4.30 and 2.13, respectively). Different samples and different specific primers of PCR in each study included may also affect the results, so we performed subgroup based on the sample and specific primer. The $H$. pylori infection rate in bile was higher in chronic cholecystitis/cholelithiasis than that in controls $(\mathrm{OR}=3.78, P=0.002)$, indicating that $H$. pylori is relatively more colonized in bile in gallstone diseases. Unfortunately, there was no significant difference in the prevalence of H. pylori infection in the gallbladder. The OR of $16 \mathrm{~S}$ rRNA was lower than that of urease gene $(2.27$ and 10.18 , respectively). Maybe false-positive result caused by urease A or $\mathrm{C}$ gene cross-reacting with the urease gene of other organisms accounted for it [40].

In addition to ethnicity and genetics, advancing age and female gender as well as obesity are also risk factors for gallstones [5]. The risk of gallstone diseases increases with age in all racial groups. Because of female sex hormones and increased cholesterol secretion, females with obesity are more likely to get gallstones [41]. However, Bohr et al. [25] found that the most significant risk factors for gallstone disease were patients with age $>65$ years and being 


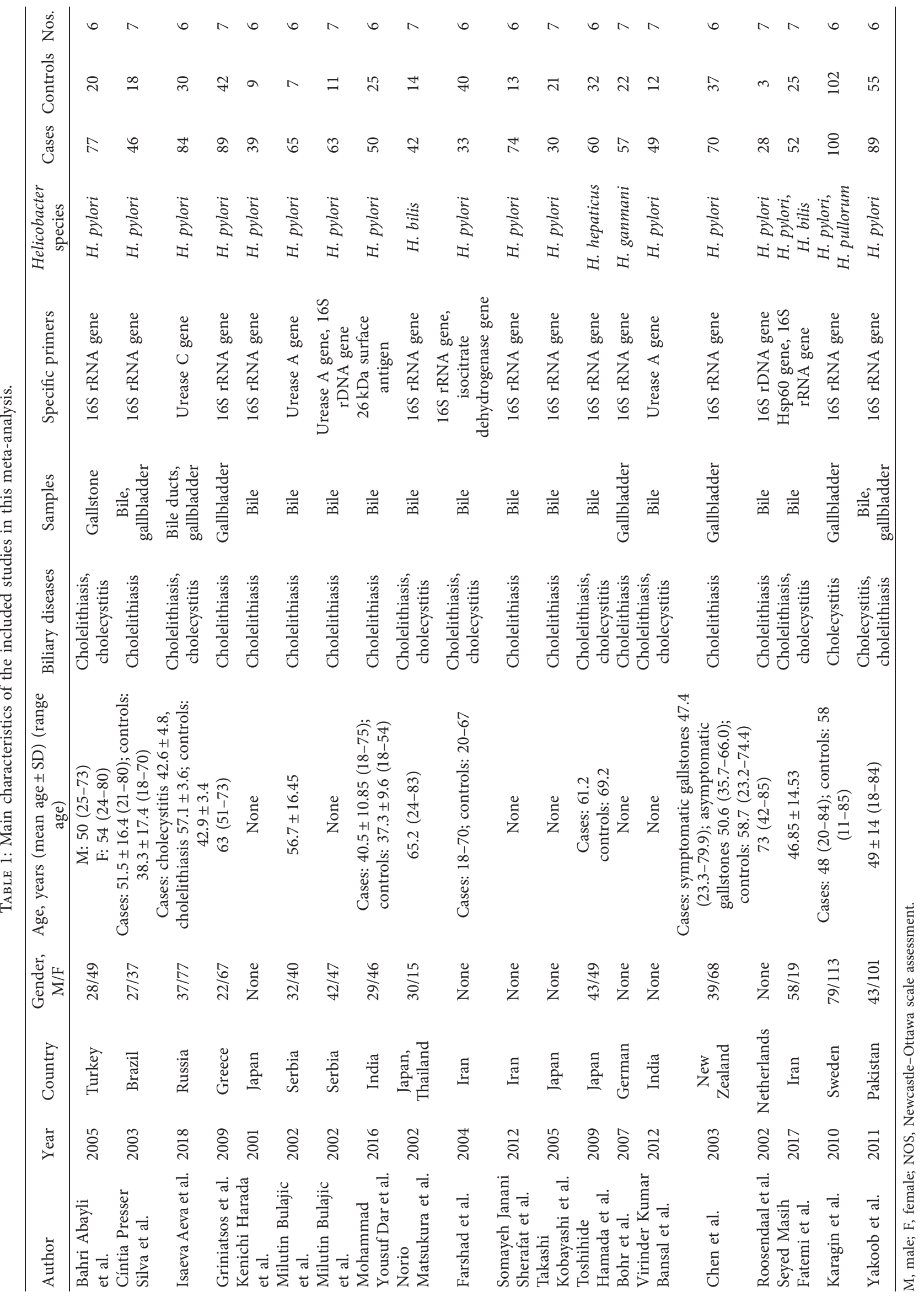




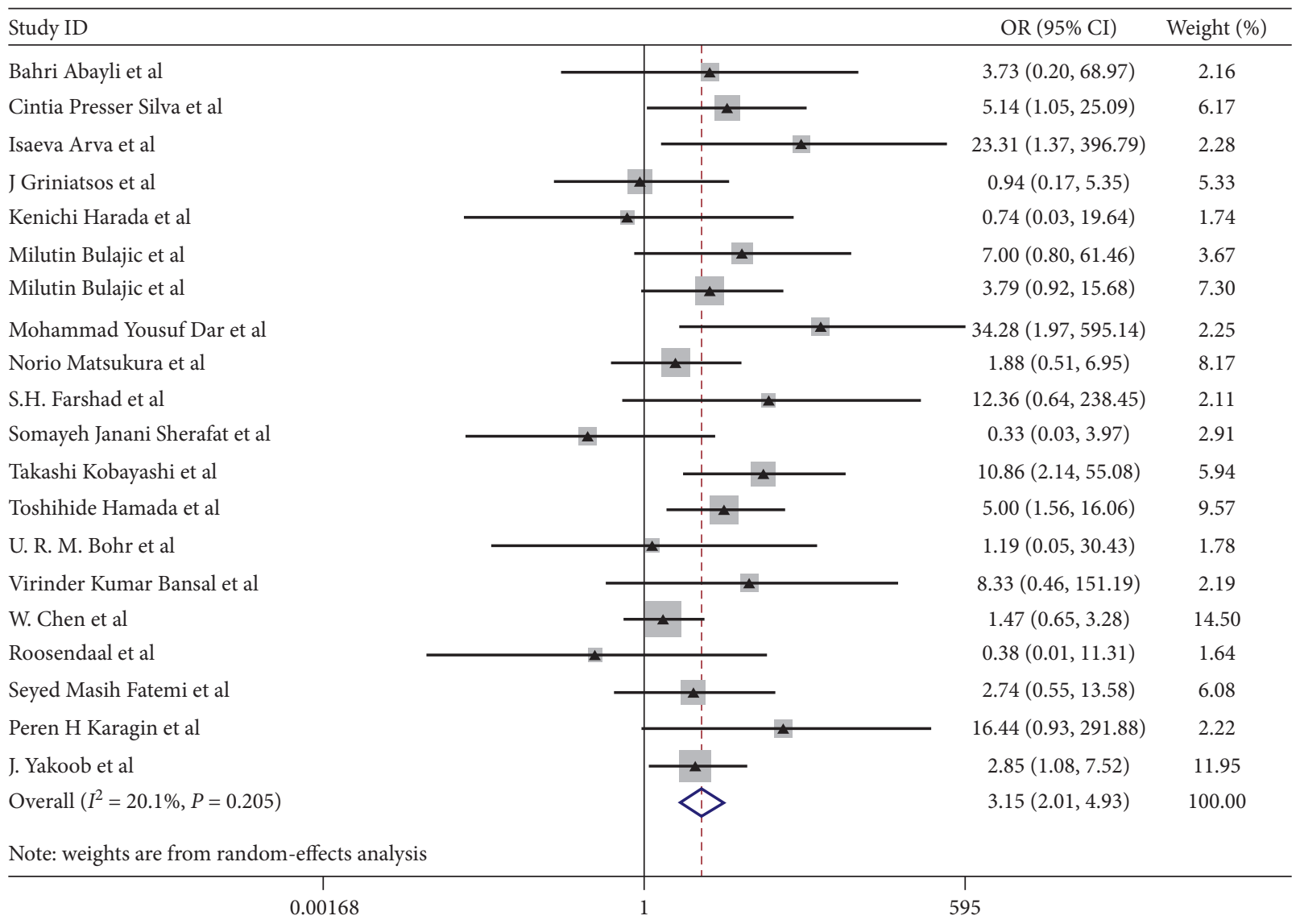

FIgURe 2: Pooled ORs for the relationship between Helicobacter species infection of the gallbladder and chronic cholecystitis and cholelithiasis. The areas of the squares are proportional to the study sample sizes.

TABLe 2: Prevalence of Helicobacter species in this meta-analysis.

\begin{tabular}{lccc}
\hline Helicobacter species & Positive in cases & Positive in controls & OR $(95 \%$ CI $)$ \\
\hline H. pylori & $246 / 1038$ & $34 / 470$ & $3.05(1.81-5.14)$ \\
H. pullorum & $6 / 100$ & $0 / 102$ & $14.10(0.78-253.71)$ \\
H. ganmani & $1 / 57$ & $0 / 22$ & $1.19(0.05-30.43)$ \\
H. hepaticus & $25 / 60$ & $4 / 32$ & $5.00(1.56-16.06)$ \\
H. bilis & $20 / 94$ & $4 / 39$ & $1.96(0.59-6.55)$ \\
\hline
\end{tabular}

OR, odds ratio; $\mathrm{CI}$, confidence interval.

overweight. Female gender was not statistically significant. Moreover, the infection rate of $H$. pylori was also associated with age [38]. Bulajic et al. [16] noted that the possibility of detecting $H$. pylori in the bile increased steadily with increasing age. High $H$. pylori infection may affect the prevalence of gallstones diseases. H. pylori infection is less related to gender. In a marching cohort conducted by Miendje Deyi et al. [42], the proportion of H. pylori infected patients was virtually the same in males and females. However, due to limited studies and insufficient data of age, gender, and body mass index (BMI), we did not evaluate their impact on chronic cholecystitis/cholelithiasis. Further studies were suggested to clarify the role of age, gender, and BMI played in gallstones diseases.

The pathways of $H$. pylori into bile have not been completely explained, one possibility is the translocation from the duodenum via Oddi's sphincter, and another is penetration via the portal circulation and lymphatic vessels $[43,44]$. Bulajic et al. [16] and Bansal et al. [30] reported that there was a strong correlation between the presence of H. pylori in the bile and in the stomach, which may testify to the above views. In a study in Thailand [45], nevertheless, they suggested that gastroduodenal and hepatobiliary infections were caused by different $H$. pylori strains based on PCR analysis.

The relationship between $H$. pylori infection and gallstones may have several potential mechanisms. Firstly, $H$. pylori can produce oxidative stress and free radical reactions through the reactive oxygen species (ROS) and reactive nitrogen species (RNS) system [46, 47] and release large amounts of proinflammatory and vasoactive substances, such as interleukins (IL)-1, IL-6, and tumor necrosis 


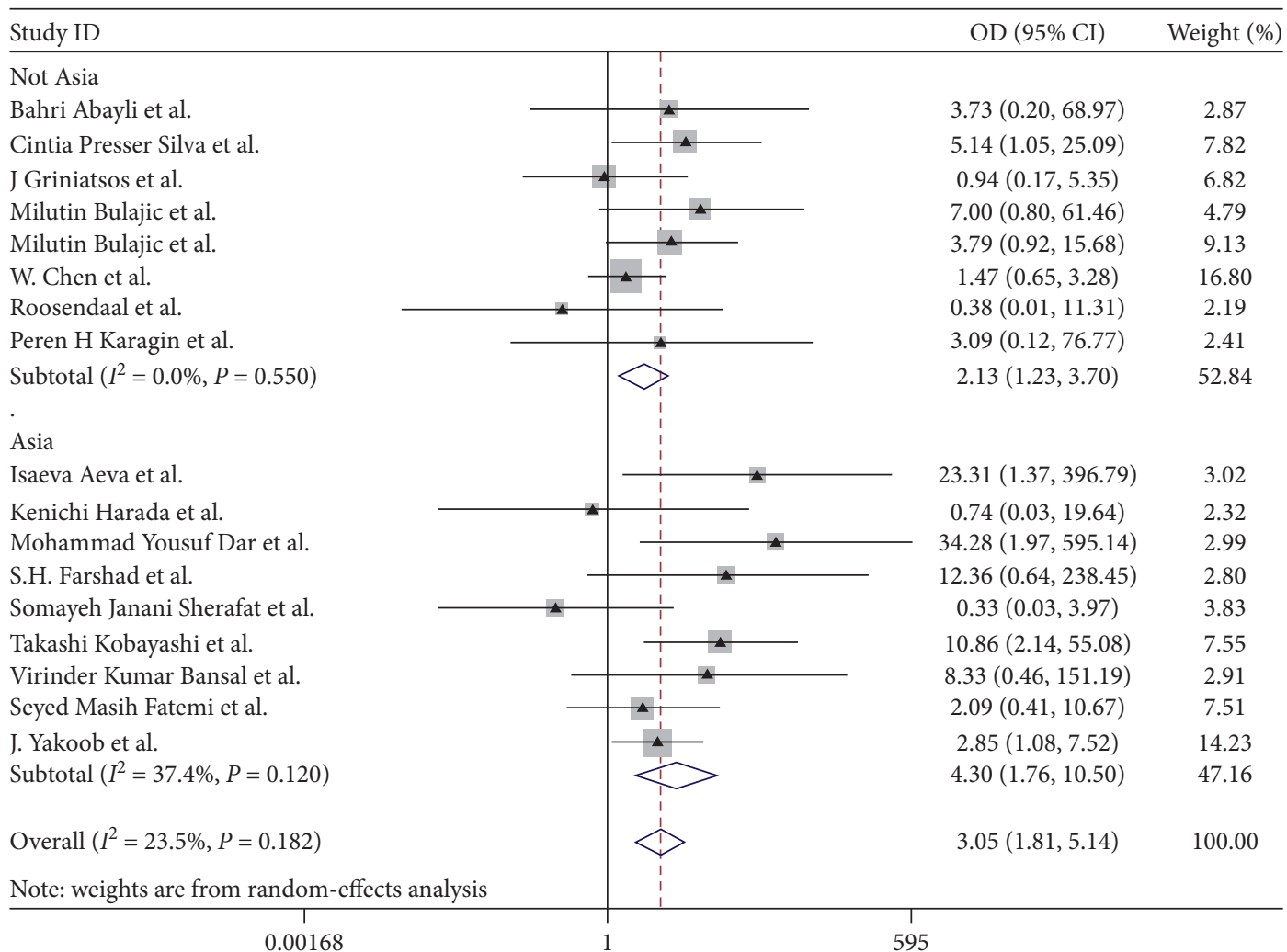

(a)

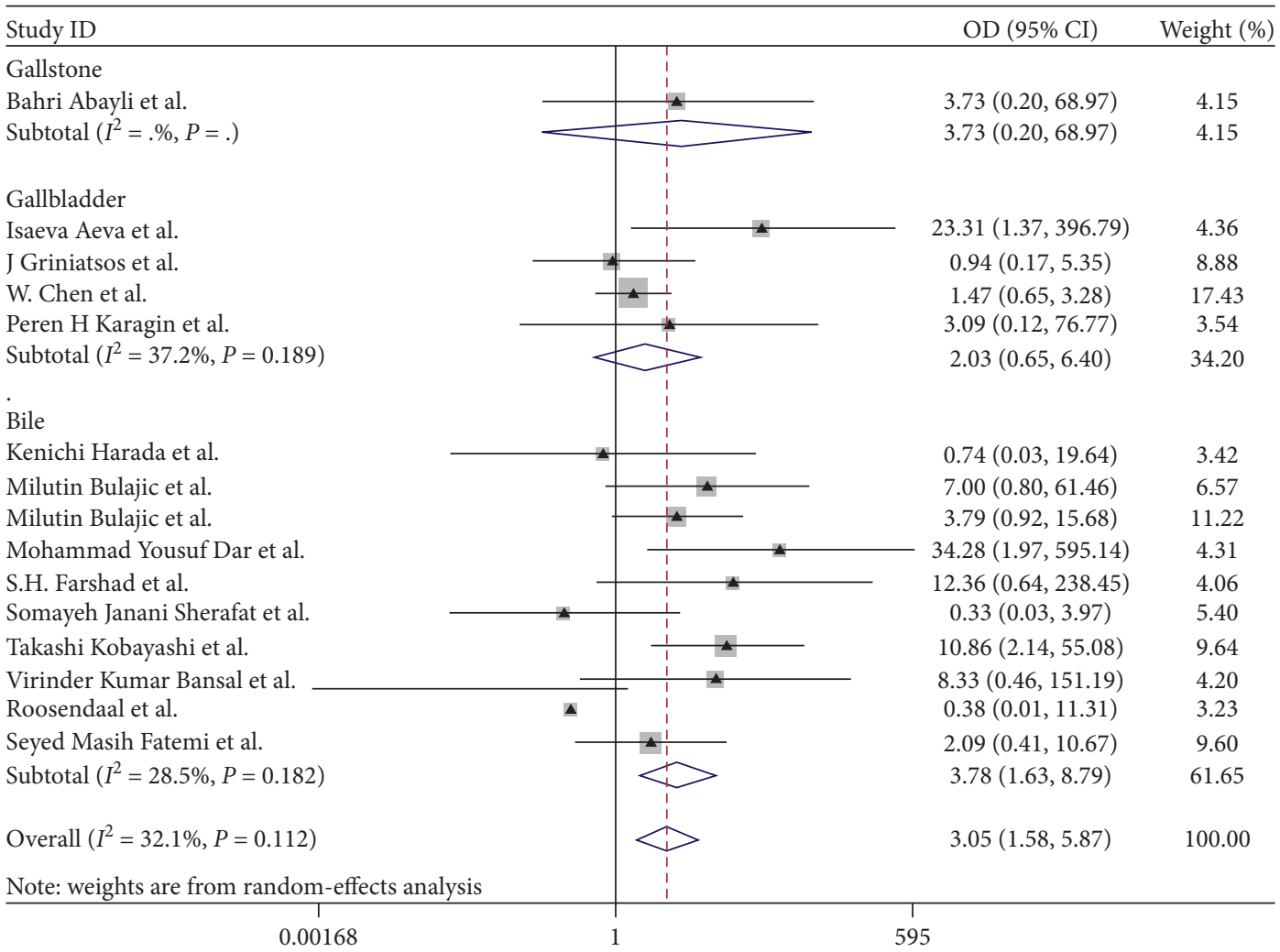

(b)

FIgUre 3: Continued. 


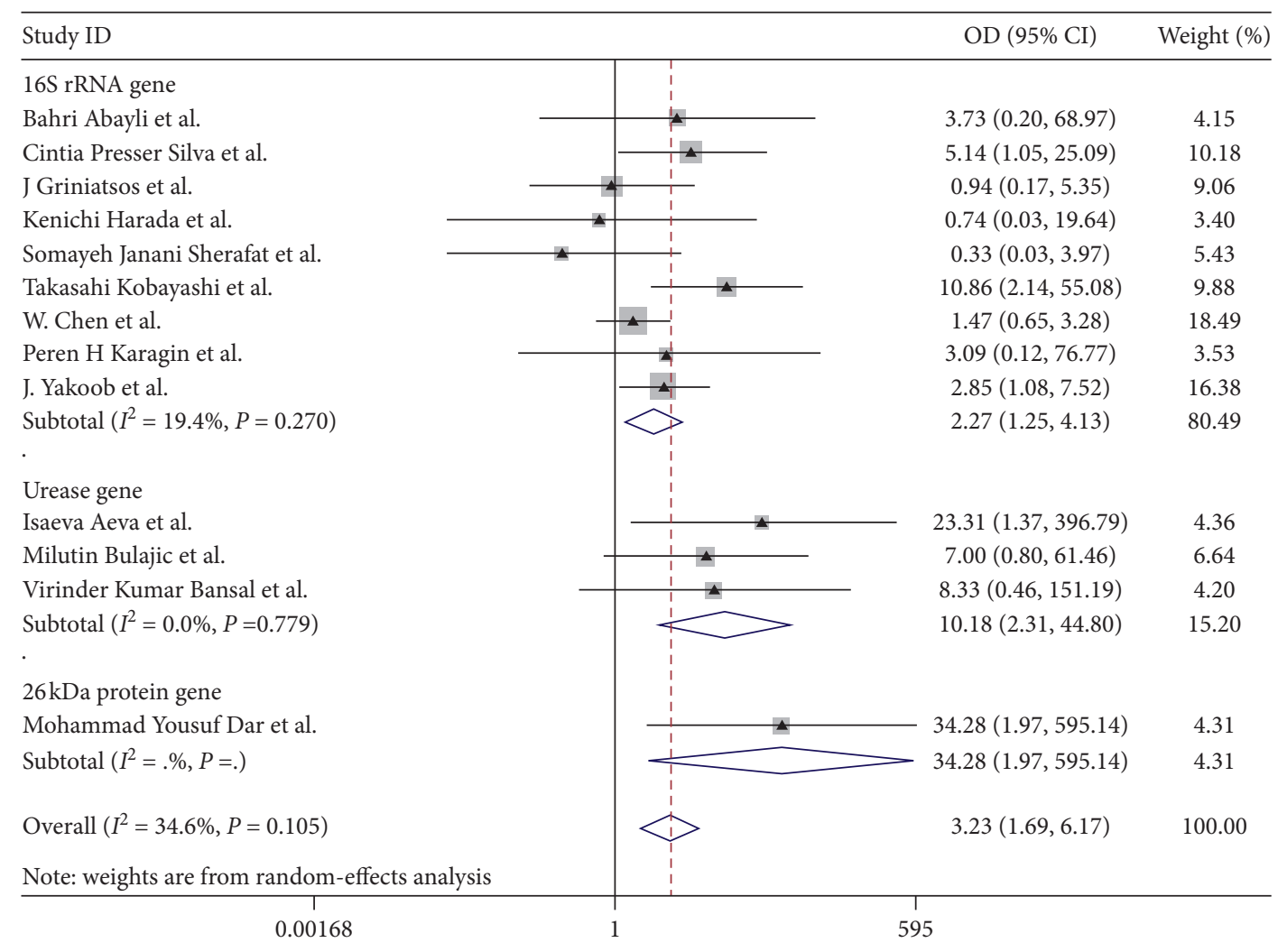

(c)

FIgURe 3: Pooled ORs for the relationship between H. pylori infection of the gallbladder and chronic cholecystitis and cholelithiasis: (a) subgroup based on country; (b) subgroup based on sample; (c) subgroup based on specific primer. The areas of the squares are proportional to the study sample sizes.

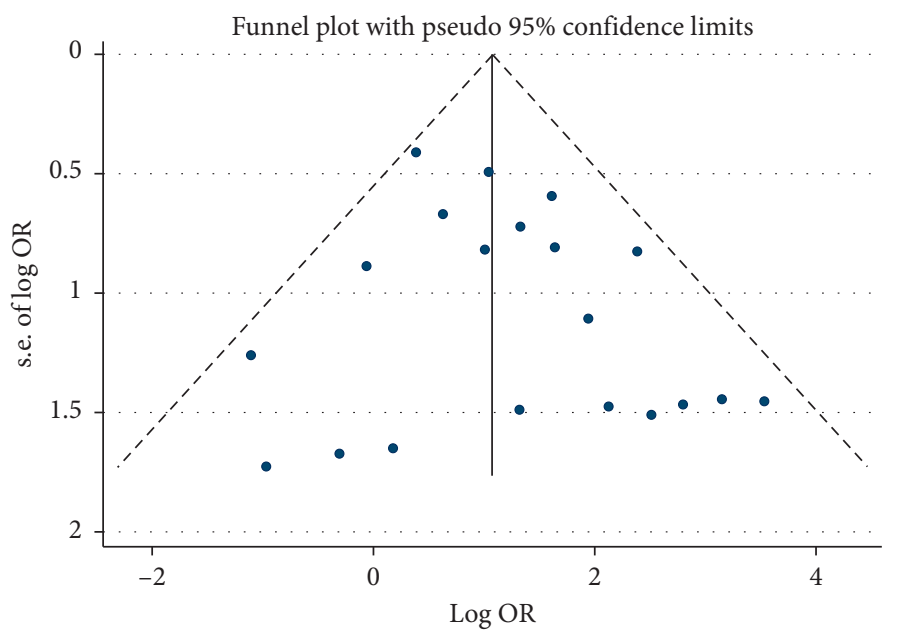

FIgure 4: Funnel plot. The distribution of dots is relatively symmetrical, suggesting no strong evidence or publication bias.

factor (TNF)- $\alpha$ [48], which are involved in gallbladder inflammatory disorders and pathogenesis of gallstones. Secondly, $H$. pylori may also promote the risk of stone formation by acting as a foreign body nidus [49].Thirdly, urease-positive Helicobacter species are capable of precipitating calcium and participating in initiating gallstone formation [50]. Fourthly, H. pylori induces beta-glucuronidase, bacterial hydrolase, and phospholipase to deconjugate bile and hydrolyze phosphatidylcholine, thus facilitating the synthesis of free bilirubin, free bile acids, and free fatty acids. Free ionized calcium ion precipitates with free bilirubin to form calcium bilirubinate. Calcium bilirubinate, free bile acids, and free fatty acids are the major components of brown pigment stones [13].

Since $H$. pylori infection promotes the formation of gallstones, we consider whether $H$. pylori eradication 
therapy can prevent the formation of gallstones. In the studies of Zhang et al. [51] and Takahashi et al. [52], they both found a statistically significant trend in the reduction of gallstones among the patients who received $H$. pylori eradication. However, the drawback is that they used the fasting ${ }^{13} \mathrm{C}$ urea breath test and serum anti- $\mathrm{Hp}$ antibody test as detection methods for $H$. pylori. Although the two methods are highly specific and sensitive, they cannot locate $H$. pylori infection in the gallbladder, which may undermine the reliability of the study. Besides, the serological test is not a reliable test for evaluating eradication therapy because antibodies can be present in the blood for a long time even after successful eradication [53]. Also, the presence of gallstones in the gallbladder was diagnosed by abdominal ultrasonography, which may lead to a false-negative result. In a word, the exact effect of eradicating $H$. pylori infection to prevent gallstone formation remains to be elucidated.

It is noteworthy that our meta-analysis also has some strengths. Firstly, in order to avoid the influence of different test methods on the research, we selected studies using PCR as the $H$. pylori infection detection method, which is a highly sensitive and specific method. Serological testing is excluded for the relatively high negative predictive value [54-56]. Besides, there was no gallbladder disease in the control group. Due to the high correlation between $H$. pylori infection and gallbladder cancer, these patients are excluded $[57,58]$. There was a previous meta-analysis which assessed the association between $H$. pylori infection and chronic cholecystitis/cholelithiasis [59]. However, the included studies mixed H. pylori and other Helicobacter species. In our study, we analyzed $H$. pylori and other Helicobacter species separately and conducted more in-depth subgroup analyses of $H$. pylori based on the extracted sample and specific primer.

However, several limitations of this meta-analysis should be noted. Most patients with cholelithiasis have been treated with antibiotics. Different doses and courses of antibiotics might inhibit the growth of the bacterium and influence the results. Besides, since the retrograde contamination is caused by ERCP, there may be false-positive results. Furthermore, there are diverse bacterial communities in human bile samples such as Pseudomonas aeruginosa, E. coli, Klebsiella pneumoniae, Enterococcus spp., and Acinetobacter spp. Tajeddin et al. [60] pointed that Escherichia coli was significantly related to gallstone formation. Our study cannot avoid interference from other bacteria. Previous studies have suggested that $H$. pylori infection may be associated with pancreatic cancer [61], liver cirrhosis, and liver cancer [62]. It is a pity that the control group could not completely exclude such patients in our study.

In conclusion, our meta-analysis showed a significant positive correlation between $H$. pylori infection in the gallbladder and increased risk of cholelithiasis and chronic cholecystitis, especially in Asian countries. The association was more pronounced using the bile sample and urease gene primer. Also, more studies are needed to determine whether age, gender, and BMI interfere with the relationship between gallbladder $H$. pylori infection and chronic cholecystitis/ cholelithiasis. It is hoped that more randomized trials in the future can better clarify the relationship between them and provide clinical evidence for the prevention and treatment of chronic cholecystitis/cholelithiasis.

$\begin{array}{ll}\text { Abbreviations } \\ \text { H. pylori: } & \text { Helicobacter pylori } \\ \text { Hp: } & \text { Helicobacter pylori } \\ \text { MALT: } & \text { Gastric malignant lymphoma of the mucosa- } \\ & \text { associated lymphoid tissue } \\ \text { PCR: } & \text { Polymerase chain reaction } \\ \text { ERCP: } & \text { Endoscopic retrograde } \\ & \text { cholangiopancreatography } \\ \text { BMI: } & \text { Body mass index } \\ \text { ROS: } & \text { Reactive oxygen species } \\ \text { RNS: } & \text { Reactive nitrogen species } \\ \text { IL: } & \text { Interleukins } \\ \text { TNF: } & \text { Tumor necrosis factor. }\end{array}$

\section{Data Availability}

The data supporting the current study are available from the corresponding author upon request.

\section{Disclosure}

This study does not include human participants or animals. Liang Wang, Junyin Chen, and Wenxi Jiang are the co-first authors.

\section{Conflicts of Interest}

All authors declare that they have no conflicts of interest.

\section{Authors' Contributions}

Wang L, Chen J, Jiang W, and Shen Z conceived and designed the study. Wang L, Chen J, Jiang W, Cen L, and Pan $J$ collected the data. Jiang W, Cen L, and Shen $Z$ analyzed the data. Wang L, Chen J, Jiang W, Cen L, Pan J, Yu C, Li Y, Chen W, Chen C, and Shen $\mathrm{Z}$ wrote the paper. Wang L, Chen J, Jiang W, Cen L, Pan J, Yu C, Li Y, Chen W, Chen C, and Shen $\mathrm{Z}$ were responsible for final approval of the paper. All authors approved the final version of the manuscript. Liang Wang, Junyin Chen, and Wenxi Jiang contributed equally.

\section{References}

[1] B. Marshall and J. R. Warren, "Unidentified curved bacilli in the stomach of patients with gastritis and peptic ulceration," The Lancet, vol. 323, no. 8390, pp. 1311-1315, 1984.

[2] N. Uemura, S. Okamoto, S. Yamamoto et al., "Helicobacter pylori infection and the development of gastric cancer," New England Journal of Medicine, vol. 345, no. 11, pp. 784-789, 2001.

[3] J. Okamoto, G. D. Friedman, D. P. Vandersteen et al., "Helicobacter pylori infection and the risk of gastric carcinoma," New England Journal of Medicine, vol. 325, no. 16, pp. 1127-1131, 1991. 
[4] S. Friedman, M. Stolte, and R. Fischer, "Helicobacter pylori gastritis and primary gastric non-Hodgkin's lymphomas," Journal of Clinical Pathology, vol. 47, no. 5, pp. 436-439, 1994.

[5] L. M. Stinton and E. A. Shaffer, "Epidemiology of gallbladder disease: cholelithiasis and cancer," Gut and Liver, vol. 6, no. 2, pp. 172-187, 2012.

[6] C. E. Ruhl and J. E. Everhart, "Gallstone disease is associated with increased mortality in the United States," Gastroenterology, vol. 140, no. 2, pp. 508-516, 2011.

[7] Y. Zheng, M. Xu, Y. Heianza et al., "Gallstone disease and increased risk of mortality: two large prospective studies in US men and women," Journal of Gastroenterology and Hepatology, vol. 33, no. 11, pp. 1925-1931, 2018.

[8] S. Ryu, Y. Chang, K. E. Yun, H.-S. Jung, J. H. Shin, and H. Shin, "Gallstones and the risk of gallbladder cancer mortality: a cohort study," American Journal of Gastroenterology, vol. 111, no. 10, pp. 1476-1487, 2016.

[9] M. Chang, T. Saito, H. Ohno et al., "Bacteria closely resembling Helicobacter pylori detected immuno histologically and genetically in resected gallbladder mucosa," Journal of Gastroenterology, vol. 31, no. 2, pp. 294-298, 1996.

[10] E. Saito, W. A. Al-Soud, I. Nilsson et al., "Helicobacter pyloriand otherHelicobacter species in gallbladder and liver of patients with chronic cholecystitis detected by immunological and molecular methods," Scandinavian Journal of Gastroenterology, vol. 40, no. 1, pp. 96-102, 2005.

[11] D.-F. Al-Soud, L. Hu, and P. Yi, "H pyloriexist in the gallbladder mucosa of patients with chronic cholecystitis," World Journal of Gastroenterology, vol. 13, no. 10, pp. 1608-1611, 2007.

[12] K. J. Maurer, M. C. Carey, and J. G. Fox, "Roles of infection, inflammation, and the immune system in cholesterol gallstone formation," Gastroenterology, vol. 136, no. 2, pp. 425-440, 2009.

[13] Y. Wang, M. Qi, C. Qin, and J. Hong, "of the biliary microbiome in gallstone disease," Expert Review of Gastroenterology \& Hepatology, vol. 12, no. 12, pp. 1193-1205, 2018.

[14] I. N. Qi and T. I. Romanova, "Gallstone disease and microbiome," Microorganisms, vol. 8, no. 6, 2020.

[15] K. Harada, S. Ozaki, N. Kono et al., "Frequent molecular identification of Campylobacter but notHelicobacter genus in bile and biliary epithelium in hepatolithiasis," The Journal of Pathology, vol. 193, no. 2, pp. 218-223, 2001.

[16] M. Bulajic, P. Maisonneuve, W. Schneider-Brachert et al., "Helicobacter pylori and the risk of benign and malignant biliary tract disease," Cancer, vol. 95, no. 9, pp. 1946-1953, 2002.

[17] M. Maisonneuve, B. Stimec, M. Milicevic et al., "Modalities of testingHelicobacter pyloriin patients with nonmalignant bile duct diseases," World Journal of Gastroenterology, vol. 8, no. 2, pp. 301-304, 2002.

[18] N. Matsukura, S. Yokomuro, S. Yamada et al., "Association between Helicobacter bilisin bile and biliary tract malignancies: H. Bilisin bile from Japanese and Thai patients with benign and malignant diseases in the biliary tract," Japanese Journal of Cancer Research, vol. 93, no. 7, pp. 842-847, 2002.

[19] R. Yokomuro, E. J. Kuipers, C. M. J. E. VandenbrouckeGrauls, and J. G. Kusters, "species are not detectable by $16 \mathrm{~S}$ rDNA PCR in bile from Dutch patients with common bile duct stones," Digestion, vol. 66, no. 2, pp. 89-91, 2002.

[20] W. Chen, D. Li, R. J. Cannan, and R. S. Stubbs, "Common presence of Helicobacter DNA in the gallbladder of patients with gallstone diseases and controls," Digestive and Liver Disease, vol. 35, no. 4, pp. 237-243, 2003.
[21] C. P. Silva, J. C. Pereira-Lima, A. G. Oliveira et al., "Association of the presence of Helicobacter in gallbladder tissue with cholelithiasis and cholecystitis," Journal of Clinical Microbiology, vol. 41, no. 12, pp. 5615-5618, 2003.

[22] S. Farshad, A. Alborzi, S. A. Malek Hosseini et al., "Identification of Helicobacter pylori DNA in Iranian patients with gallstones," Epidemiology and Infection, vol. 132, no. 6, pp. 1185-1189, 2004.

[23] B. Abayli, S. Colakoglu, M. S. Serin, and S. Erdogan, "Helicobocter pylori in the etiology of cholesterol gallstones," Journal of Clinical Gastroenterology, vol. 39, no. 2, pp. 134137, 2005.

[24] T. Kobayashi, K. Harada, K. Miwa, and Y. Nakanuma, "Helicobacter genus DNA fragments are commonly detectable in bile from patients with extrahepatic biliary diseases and associated with their pathogenesis," Digestive Diseases and Sciences, vol. 50, no. 5, pp. 862-867, 2005.

[25] U. R. M. Bohr, D. Kuester, F. Meyer et al., "Low prevalence of Helicobacteraceae in gall-stone disease and gall-bladder carcinoma in the German population," Clinical Microbiology and Infection, vol. 13, no. 5, pp. 525-531, 2007.

[26] J. Griniatsos, S. Sougioultzis, K. Giaslakiotis et al., "Does Helicobacter pylori identification in the mucosa of the gallbladder correlate with cholesterol gallstone formation?" West Indian Medical Journal, vol. 58, no. 5, pp. 428-432, 2009.

[27] T. Hamada, K. Yokota, K. Ayada et al., "Detection of Helicobacter hepaticus in human bile samples of patients with biliary disease," Helicobacter, vol. 14, no. 6, pp. 545-551, 2009.

[28] P. H. Karagin, U. Stenram, T. Wadström, and A. Ljungh, "Helicobacter species and common gut bacterial DNA in gallbladder with cholecystitis," World Journal of Gastroenterology, vol. 16, no. 38, pp. 4817-4822, 2010.

[29] J. Yakoob, M. R. Khan, Z. Abbas et al., "Helicobacter pylori: association with gall bladder disorders in Pakistan," British Journal of Biomedical Science, vol. 68, no. 2, pp. 59-64, 2011.

[30] V. Bansal, M. C. Misra, G. Chaubal et al., "Helicobacter pylori in gallbladder mucosa in patients with gallbladder disease," Indian Journal of Gastroenterology, vol. 31, no. 2, pp. 57-60, 2012.

[31] S. J. Sherafat, E. Tajeddin, M. Seyyedmajidi et al., "Lack of association between Helicobacter pylori infection and biliary tract diseases," Polish Journal of Microbiology, vol. 61, no. 4, pp. 319-322, 2012.

[32] M. Y. Dar, S. Ali, A. H. Raina et al., "Association of Helicobacter pylori with hepatobiliary stone disease, a prospective case control study," Indian Journal of Gastroenterology, vol. 35, no. 5, pp. 343-346, 2016.

[33] G. Isaeva Aeva, "Distribution of Helicobacter pylori genotypes in various sites of the hepatobiliary system," Minerva Biotecnologica, vol. 30, no. 4, pp. 102-107, 2018.

[34] S. M. Fatemi, A. Doosti, D. Shokri et al., "Is there correlation between helicobacter pylori and enterohepatic helicobacter species with gallstone cholecystitis?: 61," American Journal of Gastroenterology, vol. 112, p. S28, 2017.

[35] C. A. Fallone, S. Tran, M. Sermet, F. Discepola, M. Behr, and A. N. Barkun, "Helicobacter DNA in bile: correlation with hepato-biliary diseases," Alimentary Pharmacology \& Therapeutics, vol. 17, no. 3, pp. 453-458, 2003.

[36] D. F. Stroup, J. A. Berlin, S. C. Morton et al., "Meta-analysis of observational studies in epidemiology-a proposal for reporting," JAMA-Journal of the American Medical Association, vol. 283, no. 15, pp. 2008-2012, 2000.

[37] A. Stang, "Critical evaluation of the Newcastle-Ottawa scale for the assessment of the quality of nonrandomized studies in 
meta-analyses," European Journal of Epidemiology, vol. 25, no. 9, pp. 603-605, 2010.

[38] K. Muhsen, D. Cohen, A. Spungin-Bialik, and T. Shohat, "Seroprevalence, correlates and trends of Helicobacter pylori infection in the Israeli population," Epidemiology and Infection, vol. 140, no. 7, pp. 1207-1214, 2012.

[39] R. W. Leong and J. J. Sung, "Review article: Helicobacter species and hepatobiliary diseases," Alimentary Pharmacology \& Therapeutics, vol. 16, no. 6, pp. 1037-1045, 2002.

[40] A. Labigne, V. Cussac, and P. Courcoux, "Shuttle cloning and nucleotide sequences of Helicobacter pylori genes responsible for urease activity," Journal of Bacteriology, vol. 173, no. 6, pp. 1920-1931, 1991.

[41] E. A. Shaffer, "Epidemiology and risk factors for gallstone disease: has the paradigm changed in the 21st century?" Current Gastroenterology Reports, vol. 7, no. 2, pp. 132-140, 2005.

[42] V. Y. Miendje Deyi, J. Vanderpas, P. Bontems et al., "Marching cohort of Helicobacter pylori infection over two decades (1988-2007): combined effects of secular trend and population migration," Epidemiology and Infection, vol. 139, no. 4, pp. 572-580, 2011.

[43] A. Cariati, R. Puglisi, R. Zaffarano, F. T. Accarpio, and F. Cetta, "Helicobacter pylori and the risk of benign and malignant biliary tract disease," Cancer, vol. 98, no. 3, pp. 656-657, 2003.

[44] G. F. Helaly, E. F. El-Ghazzawi, A. H. Kazem, N. L. Dowidar, M. M. Anwar, and N. M. Attia, "Detection of Helicobacter pylori infection in Egyptian patients with chronic calcular cholecystitis," British Journal of Biomedical Science, vol. 71, no. 1, pp. 13-18, 2014.

[45] W. Boonyanugomol, N. Khuntikeo, A. Pugkhem et al., "Genetic characterization of Helicobacter pylori vacA and cagA genes in Thai gastro-duodenal and hepatobiliary patients," Journal of Infection in Developing Countries, vol. 11, no. 1, pp. 42-50, 2017.

[46] D. Zhou, W.-B. Guan, J.-D. Wang, Y. Zhang, W. Gong, and Z.-W. Quan, "A comparative study of clinicopathological features between chronic cholecystitis patients with and without Helicobacter pylori infection in gallbladder mucosa," PLoS One, vol. 8, no. 7, 2013.

[47] P. Sipos, H. Krisztina, A. Blázovics, and J. Fehér, "Cholecystitis, gallstones and free radical reactions in human gallbladder," Medical Science Monitor, vol. 7, no. 1, pp. 84-88, 2001.

[48] A. Kasprzak, A. Szmyt, A. Malkowski et al., "Analysis of immunohistochemical expression of proinflammatory cytokines (IL- $1 \alpha$, IL-6, and TNF- $\alpha$ ) in gallbladder mucosa: comparative study in acute and chronic calculous cholecystitis," Folia Morphologica (Warsz), vol. 74, no. 1, pp. 65-72, 2015.

[49] H. J. Monstein, Y. Jonsson, J. Zdolsek, and J. Svanvik, "Identification of Helicobacter pylori DNA in human cholesterol gallstones," Scandinavian Journal of Gastroenterology, vol. 37, no. 1, pp. 112-119, 2002.

[50] C. Belzer, J. G. Kusters, E. J. Kuipers, and A. H. M. Van Vliet, "Urease induced calcium precipitation by Helicobacter species may initiate gallstone formation," Gut, vol. 55, no. 11, pp. 1678-1679, 2006.

[51] F.-M. Zhang, C.-H. Yu, H.-T. Chen et al., "Helicobacter pylori infection is associated with gallstones: epidemiological survey in China," World Journal of Gastroenterology, vol. 21, no. 29, pp. 8912-8919, 2015.
[52] Y. Takahashi, N. Yamamichi, T. Shimamoto et al., "Helicobacter pylori infection is positively associated with gallstones: a large-scale cross-sectional study in Japan," Journal of Gastroenterology, vol. 49, no. 5, pp. 882-889, 2014.

[53] Y.-K. Wang, F.-C. Kuo, C.-J. Liu et al., "Diagnosis of Helicobacter pylori infection: current options and developments," World Journal of Gastroenterology, vol. 21, no. 40, pp. 11221-11235, 2015.

[54] A. A. Kerawala, N. Bakhtiar, S. Sakina Abidi, and S. Awan, "Association of gallstone and Helicobacter pylori," Journal of Medical Sciences (Peshawar), vol. 27, no. 4, pp. 269-272, 2019.

[55] M. Y. Xu, J.-H. Ma, B.-S. Yuan, J. Yin, L. Liu, and Q.-B. Lu, "Association between Helicobacter pylori infection and gallbladder diseases: a retrospective study," European Journal of Gastroenterology \& Hepatology, vol. 33, no. 6, pp. 1207-1212, 2018.

[56] T. Kim, "A cohort study on Helicobacter pylori infection and the development of gallbladder stone," Helicobacter, vol. 23, p. 90, 2018.

[57] Y. Tsuchiya, K. Mishra, V. K. Kapoor et al., "Plasma Helicobacter pylori antibody titers and Helicobacter pylori infection positivity rates in patients with gallbladder cancer or cholelithiasis: a hospital-based case-control study," Asian Pacific Organization for Cancer Prevention, vol. 19, no. 7, pp. 1911-1915, 2018.

[58] Y. Tsuchiya, E. Loza, G. Villa-Gomez et al., "Metagenomics of microbial communities in gallbladder bile from patients with gallbladder cancer or cholelithiasis," Asian Pacific Journal of Cancer Prevention, vol. 19, no. 4, pp. 961-967, 2018.

[59] L. Cen, J. Pan, B. Zhou et al., "Helicobacter pylori infection of the gallbladder and the risk of chronic cholecystitis and cholelithiasis: a systematic review and meta-analysis," Helicobacter, vol. 23, no. 1, 2018.

[60] E. Tajeddin, S. J. Sherafat, M. R. S. Majidi, M. Alebouyeh, A. H. M. Alizadeh, and M. R. Zali, "Association of diverse bacterial communities in human bile samples with biliary tract disorders: a survey using culture and polymerase chain reaction-denaturing gradient gel electrophoresis methods," European Journal of Clinical Microbiology \& Infectious Diseases, vol. 35, no. 8, pp. 1331-1339, 2016.

[61] M. Xiao, Y. Wang, and Y. Gao, "Association between Helicobacter pylori infection and pancreatic cancer development: a meta-analysis," PLoS One, vol. 8, no. 9, Article ID e75559, 2013.

[62] R. Pellicano, A. Ménard, M. Rizzetto, and F. Mégraud, "Helicobacter species and liver diseases: association or causation?" Lancet Infectious Diseases, vol. 8, no. 4, pp. 254-260, 2008. 